\title{
Low variability and absence of phenotypic correlates of Clock gene variation in a great tit Parus major population
}

\author{
Miriam Liedvogel and Ben C. Sheldon \\ M. Liedvogel (miriam.liedvogel@zooekol.lu.se) and B. C. Sheldon, Edward Grey Inst., Dept. of Zoology, Univ. of Oxford, Oxford OX1 3PS, \\ UK. ML also at: Dept. of Ecology, Lund Univ., SE-223 62 Lund, Sweden.
}

\begin{abstract}
Studies of a range of taxa, including birds, have revealed latitudinal clines in allele length at the conserved Clock locus, a gene with known influences on behaviour and physiology. Such clines might reflect adaptation to seasonal variation, a suggestion supported by a recent within-population analysis of blue tits Cyanistes caeruleus, which found associations between Clock genotype and timing of breeding in females. To test the generality of this pattern, we sequenced the polymorphic poly-Q locus of the Clock gene in 521 female great tits Parus major, which were selected based on possession of extreme breeding phenotypes. In total, we identified five alleles with one allele accounting for $96 \%$ of allelic diversity in the sample set. Overall variability at the poly-Q locus was very low, and the spatial distribution of Clock alleles across Wytham was highly homogenous. Our data further provide no evidence for a connection between Clock genotype and reproductive timing phenotype in female great tits; further, we found no effect of Clock genotype on reproductive success. Hence, these results are in contrast to the pattern found for the sympatric blue tit population inhabiting the same woodlands, suggesting that phenotypic effects of Clock are not general in passerine birds.
\end{abstract}

Characterisation of the role of genetic differences among individuals and populations in determining adaptive phenotypic differences is crucial for our understanding of evolution (Hoekstra and Coyne 2007, Ellegren and Sheldon 2008). One approach that has been taken in this field is to study the associations between candidate genes that are conserved across species and phenotypic variation (Abzhanov et al. 2004, Fitzpatrick et al. 2005, Fidler et al. 2007). The Clock gene was first identified in mice and has subsequently been characterised in a large number of animals, including several bird species. The $\mathrm{C}$ terminal portion of the Clock protein contains a variable poly-glutamine (poly-Q) motif influencing behaviour and physiology (Young and Kay 2001, Panda et al. 2002). Recent work in a blue tit population has demonstrated phenotypic correlates of variation in Clock genotype, showing females, but not males, with fewer poly-Q repeats at the variable Clock locus to breed earlier in the season (Liedvogel et al. 2009). The pattern found for within-population variation at the variable Clock locus in blue tits matches predictions derived from earlier results in birds, fish and insects, which revealed associations between Clock genotype and latitude across populations concordant with latitudinal variation in the timing of reproduction (Tauber and Kyriacou 2005, Johnsen et al. 2007, O’Malley and Banks 2008).

In order to understand the importance of single gene effects on behaviour and phenotypes in wild populations it is important to assess their generality. For example, one might compare the extent to which associations were similar at the same locus across populations (Haas et al. 2009, Korsten et al. 2010). Alternatively, for genes for which function is thought to be highly conserved, one might ask to what extent associations are consistent within sympatric populations of different species. In a previous study, we investigated the phenotypic correlates of variation at the poly-Q locus of the Clock gene within a population of blue tits Cyanistes caeruleus inhabiting Wytham Woods, near Oxford (Liedvogel et al. 2009). The data on blue tits suggest that selection in females, but not in males, for fewer poly- $\mathrm{Q}$ repeats may be operating, and the across-population associations in timing of reproduction involving this locus could be linked to intra-population variation. The present study focussed on the great tit population in Wytham, for which a great deal is known about the ecological causes and consequences of variation in timing of breeding, due to a comprehensive programme of research stretching over the last four decades (Perrins 1965, McCleery et al. 2004, Charmantier et al. 2008). Both great tits and blue tits have rather similar ecological requirements, exploit similar resources, and are under similar selection with respect to timing. Both species show similar within-year phenotypic variability in breeding phenotype (though mean first egg date in blue tits is on average 1.5 days earlier than in great tits) and for both species there is evidence of similar selection processes with respect to the timing of reproduction (Garant et al. 2007, Liedvogel et al. 2009).

The main objectives of this work were to characterise the poly-Q locus of the Clock gene in female great tits with 
extreme breeding phenotypes and investigate genetic variability in the target region to determine how this variation relates, if at all, to the phenotypes of individuals, and finally measure the selective consequences of that variation. So far nothing is known about the generality of the latitudinal cline, or the within-population association between Clock genotype and breeding phenotype in the great tit. Complementing the previously obtained findings for the blue tit population with data from the sympatric great tits allows us to assess the generality of the patterns seen; if the patterns we see are general and reflect conserved underlying biochemical pathways, then we expect to see the same patterns of variation in both species.

\section{Methods}

\section{Study population and sampling}

We used data and blood samples obtained as part of an ongoing study on the breeding ecology and haematozoa of great tits in Wytham Woods, Oxfordshire, UK $\left(51^{\circ} 47^{\prime} \mathrm{N}\right.$, $\left.1^{\circ} 20^{\prime} \mathrm{W}\right)$. All breeding attempts of great tits in nestboxes (approximately $250-450$ breeding pairs) are monitored from nest-building until all nestlings have fledged, by regular visits to each nestbox. Parents are caught and identified, and nestlings are ringed on day 15 after hatching (hatch day $=$ day 1 , where hatch date is defined as the date of first hatching in cases of asynchronous hatching). Since 2004, blood samples of the entire breeding population have been collected by brachial vene-puncture, under licence, and stored in Ethanol or SET buffer $(0.15 \mathrm{M} \mathrm{NaCl}, 1 \mathrm{mM}$ EDTA, 50 mM Tris-HC1, pH 8.0). All blood samples used in this study were collected during the breeding seasons between 2004 and 2008 (between day seven and day 14 of the nestling phase). Genomic DNA was extracted using a standard ammonium acetate protocol, and purified DNA was stored at $-20^{\circ} \mathrm{C}$.

\section{Phenotype selection criteria}

In this study, we analysed blood samples from great tit female breeders between 2004 and 2008 with extreme breeding phenotypes (either early or late). Selection criteria for extreme breeding phenotypes were based on upper and lower quartiles of standardized values for lay date (LD) (standardized values were calculated as $\left(\mathrm{LD}_{\text {stand }}=(\mathrm{LD}-\right.$ $\left.\mathrm{LD}_{\text {(average/year) }}\right) / \mathrm{SD}$ ) with $\mathrm{SD}$ as the standard deviation of LD per year). To select birds with extreme breeding phenotypes we used two approaches: 1) each breeding event (including multiple breeders more than once), and 2) each female great tit breeder was individually scored as being very early or very late, where single values (for birds, that bred only once between 2004-2008), or average values over all breeding years (for multiple breeders), were taken as relevant measures. 'Extreme phenotypes' were defined as breeding attempts, and/or individual values falling in the upper or lower quartile. Birds $(n=521)$ that were categorised as extreme phenotypes in at least one of the two selection methods were genotyped at the poly-Q locus of the Clock gene (mean differences (SE) in standardized first egg date between the two groups of birds so formed: 1.039
(0.042) upper quartile; -0.927 (0.040) lower quartile) Of the 521 genotyped individuals, $199(38.2 \%)$ bred more than once in the focal period 2004 to 2008: 135 female great tits bred twice, 45 bred three times, 18 individuals bred four times and one individual bred successfully in all five years. The full dataset (including multiple breeders) covered 804 breeding events. In addition, and as a control for our female dataset selected for extreme phenotypes, we analysed Clock gene variability at the poly-Q locus of randomly selected (with respect to phenotype) samples from 55 females and 36 male breeders.

\section{Clock poly-Q characterisation}

Genomic DNA samples were sequenced at the poly-Q locus of the Clock gene to characterise the allelic variation at the trinucleotide tandem repeat locus, which has been shown to be polymorphic in several bird species (chicken: Larkin et al. 1999; barn owl: Fidler and Gwinner 2003; bluethroat: Johnsen et al. 2007; blue tit: Johnsen et al. 2007, Liedvogel et al. 2009, Steinmeyer et al. 2009; blackcap: Steinmeyer et al. 2009). We characterised the glutamine rich tandem repeat locus of the Clock gene for 225 great tit females with extreme breeding phenotypes by PCR amplification using the primer set previously described for sequencing of the Clock poly-Q allele in blue tits (Johnsen et al. 2007). This primer set has further been shown to successfully amplify the poly-Q Clock locus in another songbird species, the blackcap (Steinmeyer et al. 2009). PCR protocol and temperature profiles were adapted according to Johnsen et al. 2007 in a $10 \mu \mathrm{l}$ reaction volume. Amplified products were prepared for sequencing using Qiagen MinElute 96 UF PCR Purification Kits and QiaVac Multiwell vacuum manifolds. Nucleotide sequences of purified PCR fragments were determined with the BigDye Terminator ready reaction mix, ver. 3.1 under standard sequencing conditions according to the manufacturer's protocol. Unincorporated dye terminators were removed by isopropanol precipitation. Reaction products were detected on an ABI PRISM genetic analyser 3100, and edited using Sequencher 4.8. To determine the Clock genotype of all selected 521 females with extreme breeding phenotypes on a larger scale, we adapted the 6-FAM labelled primer combination design and amplification protocol as described in Johnsen et al. (2007). Amplification products $(2 \mu \mathrm{l})$ were resolved using an ABI PRISM genetic analyzer 3100 and a molecular size standard. Allele sizes were resolved by GeneMapper 3.7 alongside with three positive control samples of known Clock genotype (number of tandem repeats was determined by sequencing). We succeeded in genotyping all 612 great tit samples. To assess the repeatability of the genotyping protocol, we analysed different samples from the same individuals (multiple breeders) collected over subsequent years (ten individuals; three samples per individual), the results agreed in all cases. To confirm the comparability of sequencing and genotyping reaction, all samples for which Clock genotype was characterised by sequencing reaction $(\mathrm{n}=225)$ were also analysed by genotyping reaction, resulting in $100 \%$ consistency. Hence both methods used to determine Clock genotype in this study reveal a high level of reliability of the applied methods. 
Sequence identities were determined by comparison with homologous sequences present on GenBank using WU-BLAST 2 (<http://www.ebi.ac.uk/Tools/blast2/>). Sequence alignment was constructed using ClustalW (Thompson et al. 1994).

\section{Ecological genetic analysis}

Calculation of allelic frequency data and tests for deviation from Hardy-Weinberg equilibrium (HWE) were performed using Genepop v. 4.0 (Raymond and Rousset 1995) with the following Markov chain parameters: dememorisation 10 000; batches 10 000; iterations per batch 10000 .

\section{Defining clock genotype}

A phenotypic correlation of Clock gene variation with breeding phenotype has been found for female blue tits inhabiting the same woodlands as the great tits analysed in this study (Liedvogel et al. 2009). To investigate the generality of this pattern, we analysed the Clock gene variation in the great tit for phenotypic correlation with breeding type in a similar manner. As in the previous study, we defined poly-Q Clock genotype as the sum of poly-Q repeats of both alleles $(\mathrm{p}+\mathrm{q})$ (Liedvogel et al. 2009). To facilitate comparison with the study on blue tits (Liedvogel et al. 2009), we analysed the data with Clock genotypes classified in three categories of allele length, grouped (agreeable with Johnsen et al. 2007) as follows: 1) heterozygous birds with one allele smaller than $\mathrm{Q}_{14}$ and the other allele $\mathrm{Q}_{14}$ or longer $\left.\left(<\mathrm{Q}_{14} / \geq \mathrm{Q}_{14}\right)(\mathrm{n}=36), 2\right)$ birds homozygous for the most common allele $\left(\mathrm{Q}_{14}\right)(\mathrm{n}=$ 480), and 3) heterozygous birds with one allele $Q_{14}$ and the other allele longer than $\mathrm{Q}_{14}\left(\geq \mathrm{Q}_{14} />\mathrm{Q}_{14}\right)(\mathrm{n}=5)$. Both genotype classification methods revealed similar results, and as in Liedvogel et al. (2009) we opted for the use of a continuous variable representing Clock poly-Q genotypes. To facilitate comparison between great tits and blue tits (Johnsen et al. 2007, Liedvogel et al. 2009) we used the genotype categories defined here for graphical representation of associations between genotype and timing of breeding.

\section{Clock genotype and timing of breeding}

We explored the relationship between Clock genotype at the variable poly-Q locus and timing of breeding using general linear mixed models (GLMM) with normal error distribution and identity links, while correcting for appropriate fixed (age, sex, altitude, breeding density), and random (ring number to account for repeated measures of individuals between years) effects based on the knowledge developed over the course of the population studies at Wytham (for further details see Liedvogel et al. 2009, all parameters and effects used in the models applied here are identical, unless stated otherwise). We tested for associations between Clock genotype and the following aspects of reproductive scheduling, which together summarise important aspects of reproductive timing phenotype: 1) lay date, i.e. date of laying the first egg (LD); 2) observed hatch date
$(\mathrm{OH})$; 3) incubation duration (ID). Timing parameters were standardized for each year as described.

\section{Clock genotype and environmental variation}

To test whether the distribution of specific alleles in the great tit population varies as a function of the environment, and whether any patterns detected are related to changes in the timing of breeding, we modelled Clock genotypic variation as a function of the following environmental variables known to be associated with variation in reproductive scheduling in great tits: altitude, oak richness (measured as number of oaks within $75 \mathrm{~m}$ radius) and breeding density (calculated per year per woodland sector, Liedvogel et al. 2009).

\section{Clock genotype and fitness measures}

We estimated selection on the polymorphic locus of the Clock gene directly, by regressing fecundity-related fitness measures (specifically: number of fledglings, number of recruits, and number of grand recruits (in total, and via daughters and sons separately) on genotypic values of individuals. We calculated standardized selection differentials measuring the effect of genotype on fitness by regressing fitness (relative number of fledged individuals, calculated as number of individuals fledged for a breeding event divided by the mean fitness of the population) on the standardized value of Clock genotype (individual valuemean/SD).

Analyses were carried out using GenStat 11 (VSN International Ltd).

\section{Results}

\section{Characterisation of the great tit poly-Q locus}

We identified five great tit poly-Q Clock alleles in the Wytham great tits $(\mathrm{n}=521)$ that differed in length by increments of Q-encoding CAG repeats. An alignment of the predicted protein sequences with the corresponding blue tit (DQ026515) and bluethroat (AY905385) orthologues is shown in Fig. 1. Sequence information for each Clock allele was obtained from a minimum of two representative great tits, amplified sequences were confirmed as Clock orthologues, and nucleotide sequences were deposited with GenBank (GenBank acc. no. GQ847506GQ847510). At the nucleotide level, all great tit alleles are related by poly-Q encoding CAG repeats (Fig. 1). The composition of CAG and CAA (both poly-Q coding) repeats within the variable tandem repeats region differed slightly between bluethroat, blue tits and great tits, but is highly consistent within each species (Fidler and Gwinner 2003). The poly-Q flanking sequence in great tits is $100 \%$ identical with the blue tit and bluethroat sequences, and this region in general is highly homologous within Aves (Fidler and Gwinner 2003). 


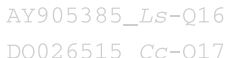

GQ847510_Pm-Q15

GQ847509_Pm-Q14

GQ847508_Pm-Q13

GQ847507_Pm-Q12

GQ847506 Pm-Q11

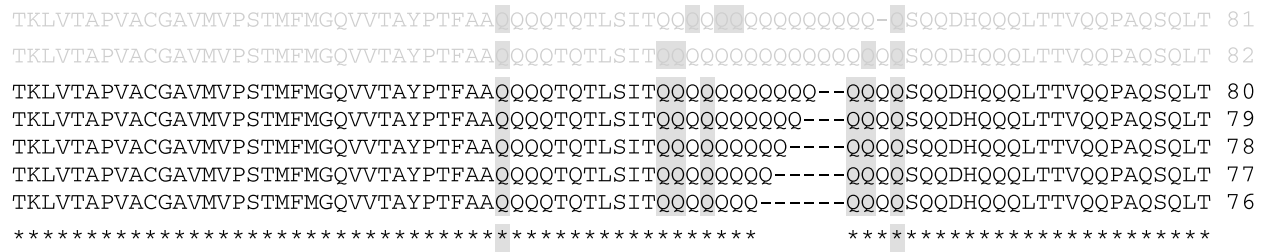

Figure 1. Comparative alignment of identified great tit Clock alleles around the poly-Q with published blue tit and bluethroat sequence (alleles with the highest number of poly-Q repeats from published sequences were chosen as reference). The predicted protein sequences of great tit poly-Q alleles identified here only differ in the number of CAG repeats at the variable Clock poly-Q locus; sequence flanking this variable locus is identical. Qs highlighted in grey depict glutamine residues encoded for by the triplet CAA. GenBank accession no. associated with the sequences are shown; suffixes correspond to the species (Ls=Luscinia svecica; $C c=C y a n i s t e s$ caeruleus; Pm $=$ Parus major) and number of poly-Q repeats.

\section{Within-population allelic variation in the great tit}

We determined Clock allele frequencies for 521 great tit females with extreme breeding phenotypes (Table 1). The level of genetic variability at the poly-Q locus of the Clock allele was very low: mean observed heterozygosity $\mathrm{H}$ of the great tit population was 0.077 . The low level of heterozygosity reflects one single great tit allele $\left(Q_{14}, 14\right.$ poly-Q repeats) being much more abundant than the other four alleles; this common allele accounts for $96 \%$ of the Wytham population. Mean observed heterozygosity for both subsets of male $(\mathrm{H}=0.0909)$ and female $(\mathrm{H}=0.0027)$ controls (randomly selected breeding phenotypes) is similarly low as for the group of females with selected breeding phenotypes. The great tit population deviated slightly, but significantly, from HWE at the poly-Q locus of the Clock gene ( $\mathrm{p}=$ 0.009 ) displaying a deficiency of heterozygotes (expected/ observed no. homozygotes $=477.33 / 481$; expected $/$ observed no. heterozygotes $=43.67 / 40)$, but the overall allelic diversity at the variable locus is very low when compared with within-population variation in other passerines (Fidler et al. 2007, Liedvogel et al. 2009, Steinmeyer et al. 2009).

\section{Clock genotype, timing phenotype and environmental variation}

We did not find an association between Clock poly-Q genotype and timing parameters in female great tits (supplemental Fig. S1; p $>0.535$, for details of the full models, including all fixed effects, supplemental Table S1). To test for an association between environmental and genetic variation in the great tit population, we analysed the spatial distribution of Clock genotype across Wytham. Given the result that Clock gene variability in the Wytham great tit population (female breeders) is generally very low, we would expect Clock alleles to be homogenously distributed across the woodlands. Spatial distribution of Clock genotype did not vary with respect to altitude, oak tree abundance (within a $75 \mathrm{~m}$ radius) or breeding density as measures of environmental heterogeneity $(p>0.141)$. We further found no evidence for any genotype $\times$ environment interaction with respect to reproductive scheduling. We analysed the data using the same model as described in supplemental Table S1 (age, altitude, breeding density included as fixed effects; ring number fitted as random effect) plus the relevant interactions, fitting the interaction between Clock genotype and each measure for environmental heterogeneity as selected above (all interactions n.s.: Clock $\times$ altitude: $\mathrm{p}>0.565$; Clock $\times$ oak richness: $\mathrm{p}>0.102$; Clock $\times$ breeding density: $\mathrm{p}>0.471)$. No small-scale correlation between Clock genotype and environmental parameter is present in the Wytham great tit population (female breeders only), analysed here.

\section{Clock genotype and fitness measures}

We found no evidence for natural selection on allelic variants. There was no effect of Clock genotype on the number of fledged young, the number of recruits or the number of grand recruits (separately analysed for grand recruits via daughters and sons; $p>0.293$ ), data on the full model with the number of fledglings per breeding event as fitness measure are shown in supplemental Table S2 and Fig. S2.

\section{Discussion}

The glutamine-rich region of the Clock gene is highly conserved and of variable length in different species (for an across-taxa comparative analysis see Saleem et al. 2001) and highly homogeneous within Aves (Fidler and Gwinner 2003). Evidence for a latitudinal cline in mean poly-Q Clock repeat length (with longer alleles associated with higher latitudes) has been found in blue tits, but not in bluethroats (Johnsen et al. 2007). A latitudinal cline in the same direction as obtained in the blue tits was also found in Chinook salmon Oncorhynchus tshawytscha (O'Malley and Banks 2008). Together these data suggest an adaptation to ecological factors correlated with latitude such as photoperiod.

Recent data on a within-population study of Clock gene variation in blue tits further suggest that the latitudinal across-population associations involving this variable locus could be linked to intra-population variation with respect to the timing of reproduction. Clock genotypes with varying length of the poly-Q locus have been shown to correlate with timing parameters in female, but not in male blue tits of the Wytham population: fewer poly-Q repeats were associated with shorter incubation duration, earlier lay date or observed hatch date (Liedvogel et al. 2009). Here, we analysed selected female great tits with extreme breeding phenotypes, to enhance the power of our analysis. The great tit population inhabits the same woodlands as the blue tit population studied earlier, which allows us to supplement 
the great tit dataset with blue tit information on poly-Q Clock genotype readily available and test the generality of the pattern of phenotypic correlates of Clock gene variation found in the blue tit population. If the identified pattern in the blue tit reflects a conserved underlying biochemical pathway then we expected the same patterns of variation in both species. However, overall variability at the polymorphic locus in the sample set of female great tits analysed here was very low, reflecting one allele $\left(\mathrm{Q}_{14}\right)$ accounting for $96 \%$ of the great tit alleles identified. This finding is in contrast to the within-population blue tit Clock gene variability (Table 1), where the mean level of heterozygosity was 0.565 (the most common allele in the blue tit was $\mathrm{Q}_{12}$, accounting for $60 \%$ of the allelic variation). Allelic variability in bluethroats analysed in the cross-population study (Johnsen et al. 2007) was also lower than in the blue tit populations analysed in the same study (12 bluethroat populations; mean observed heterozygosity $=0.213$, one monomorphic population, otherwise $\mathrm{H}$ between 0.048 and 0.476). Bluethroats, unlike blue tits, did not show any correlation of allelic variation in the Clock gene with latitude.

The fact that the (variable) poly-Q motive is highly conserved in many organisms is indicative of a functional role of the Clock protein. In vitro studies have shown that deletion of part of the poly-Q region resulted in reduced transcription activation efficiency of the protein, demonstrating that the trinucleotide tandem repeat is important for Clock protein function (Darlington et al. 1998). As longer tandem repeats are more likely to be polymorphic, an increase in repeat number would result in the variable locus exhibiting a greater degree of polymorphism. The fact that we do not see an enhanced degree of polymorphism in the great tit samples analysed might be due to the fact that CAG-repeat variation at the poly-Q locus is tightly regulated and selection acts in favour of $\mathrm{Q}_{14}$ in this species.

What can we conclude about the generality of an association between Clock gene variability and breeding phenotype? Our analyses on female great tits of the Wytham population do not confirm the association between breeding phenotype and Clock genotype we found in female blue tits of the population inhabiting the same woodland. If the pattern found in blue tits reflects a conserved underlying biochemical pathway, we would expect the pattern to be general; this is clearly not the case. Two further interesting questions remain: First, whether great tit populations in general show a lower degree of variability at the polymorphous Clock locus, or if the pattern found here is specific for this population. Second, comparisons of latitudinal clines in allele frequency in the great tit would help to establish whether variation at this locus may have played a role in adaptation to variable environments (Johnsen et al. 2007). Comparative studies of variation at this locus across the species' latitudinal range would be an important next step.

In summary, we identified five Clock alleles in great tits, varying in poly-Q repeat lengths at the polymorphic locus, with one allele accounting for $96 \%$ allelic diversity. Among female great tits of the focal population there is no evidence for an association between poly-Q Clock genotype and breeding phenotype, in contrast to the finding for sympatric female blue tits. Our data suggest that within population 
associations between Clock genotype and breeding phenotype are not general across species.

Acknowledgements - This work was supported by a Marie Curie Intra-European Fellowship (MEIF-CT-2006-040639) to ML, and by NERC and BBSRC grants to BCS. We thank all Wytham fieldworkers collecting breeding data and blood samples over many years that made this study possible.

\section{References}

Abzhanov, A., Protas, M., Grant, B. R., Grant, P. R. and Tabin C. J. 2004. Bmp4 and morphological variation of beaks in Darwin's finches. - Science 305: 1462-1465.

Charmantier, A., McCleery, R. H., Cole, L. R., Perrins, C., Kruuk, L. E. B. and Sheldon, B. C. 2008. Adaptive phenotypic plasticity in response to climate change in a wild bird population. - Science 320: 800-803.

Darlington, T. K., Wager-Smith, K., Ceriani, M. F., Staknis, D., Gekakis, N., Steeves, T. D. L., Weitz, C. J., Takashashi, J. S. and Kay, S. A. 1998. Closing the circadian loop: CLOCKinduced transcription of its own inhibitors per and tim. - Science 280: 1599-1603.

Ellegren, H. and Sheldon, B. C. 2008. Genetic basis for fitness differences in natural populations. - Nature 452: 169-175.

Fidler, A. and Gwinner, E. 2003. Comparative analysis of avian BMAL1 and CLOCK protein sequences: a search for features associated with owl nocturnal behaviour. - Comp. Biochem. Physiol. B Biochem. Mol. Biol. 136: 861-874.

Fidler, A. E., van Oers, K., Drent, P. J., Kuhn, S., Mueller, J. C. and Kempenaers, B. 2007. DRD4 gene polymorphisms are associated with personality variation in a passerine bird. - Proc. R. Soc. Lond. B 274: 1685-1691.

Fitzpatrick, M. J., Ben Shahar, Y., Smid, H. M., Vet, L. E. M., Robinson, G. E. and Sokolowski, M. B. 2005. Candidate genes for behavioural ecology. - Trends Ecol. Evol. 20: 96104.

Garant, D., Kruuk, L. E. B., McCleery, R. H. and Sheldon, B. C. 2007. The effects of environmental heterogeneity on multivariate selection on reproductive traits in female great tits. - Evolution 61: 1546-1559.

Haas, F., Pointer, M. A., Saino, N., Brodin, A., Mundy, N. I. and Hansson, B. 2009. An analysis of population genetic differentiation and genotype-phenotype association across the hybrid zone of carrion and hooded crows using microsatellites and MC1R. - Mol. Ecol. 18: 294-305.

Hoekstra, H. E. and Coyne, J. A. 2007. The locus of evolution: evo devo and the genetics of adaptation. - Evol. Int. J. Org. Evol. 61: 995-1016.
Johnsen, A., Fidler, A. E., Kuhn, S., Carter, K. L., Hoffmann, A., Barr, I. R., Biard, C., Charmantier, A., Eens, M., Korsten, P., Siitari, H., Tomiuk, J. and Kempenaers, B. 2007. Avian Clock gene polymorphism: evidence for a latitudinal cline in allele frequencies. - Mol. Ecol. 16: 4867-4880.

Korsten, P., Mueller, J. C., Hermannstädter, C., Bouwman K. M., Dingemanse, N. J., Drent, P. J., Liedvogel, M., Matthysen, E., van Oers, K., van Overveld, T., Patrick S. C., Quinn, J. L., Sheldon, B. C., Tinbergen, J. M. and Kempenaers, B. 2010. Association between DRD4 gene polymorphism and personality variation in great tits: a test across four wild populations. - Mol. Ecol. 19: 832-843.

Larkin, P., Baehr, W. and Semple-Rowland, S. L. 1999. Circadian regulation of iodopsin and clock is altered in the retinal degeneration chicken retina. - Mol. Brain Res. 79: 253-263.

Liedvogel, M., Szulkin, M., Knowles, S. C. L., Wood, M. J. and Sheldon, B. C. 2009. Phenotypic correlates of Clock gene variation in a wild blue tit population: evidence for a role in seasonal timing of reproduction. - Mol. Ecol. 18: 2444-2456.

McCleery, R. H., Pettifor, R. A., Armbruster, P., Meyer, K., Sheldon, B. C. and Perrins, C. M. 2004. Components of variance underlying fitness in a natural population of the great tit Parus major. - Am. Nat. 164: E62-E72.

O'Malley, K. G. and Banks, M. A. 2008. A latitudinal cline in the Chinook salmon Oncorhynchus tshawytscha Clock gene: evidence for selection on PolyQ length variants. - Proc. R. Soc. Lond. B 275: 2813-2821.

Panda, S., Hogenesch, J. B. and Kay, S. A. 2002. Circadian rhythms from flies to human. - Nature 417: 329-335.

Raymond, M. and Rousset, F. 1995. GenePop, ver. 1.2: population genetics software for exact tests and ecumenicism. - J. Heredity 86: 248-249.

Perrins, C. M. 1965. Population fluctuations and clutch-size in the great tit, Parus major L. - J. Anim. Ecol. 34: 601-647.

Saleem, Q., Anand, A., Jain, S. and Brahmachari, S. K. 2001. The polyglutamine motif is highly conserved at the Clock locus in various organisms and is not polymorphic in humans. - Hum. Genet. 109: 136-142.

Steinmeyer, C., Mueller, J. C. and Kempenaers, B. 2009. Search for informative polymorphisms in candidate genes: clock genes and circadian behaviour in blue tits. - Genetica 136: 109-117.

Tauber, E. and Kyriacou, C. P. 2005. Molecular evolution and population genetics of circadian clock genes. - Methods Enzymol. 393: 797-817.

Thompson, J. D., Higgins, D. G. and Gibson, T. J. 1994. CLUSTAL W: improving the sensitivity of progressive multiple sequence alignment through sequence weighting, positionspecific gap penalties and weight matrix choice. - Nucl. Acids Res. 22: 4673-4680.

Young, M. W. and Kay, S. A. 2001. Time zones: a comparative genetics of circadian clocks. - Nature Rev. Gen. 2: 702-715. 

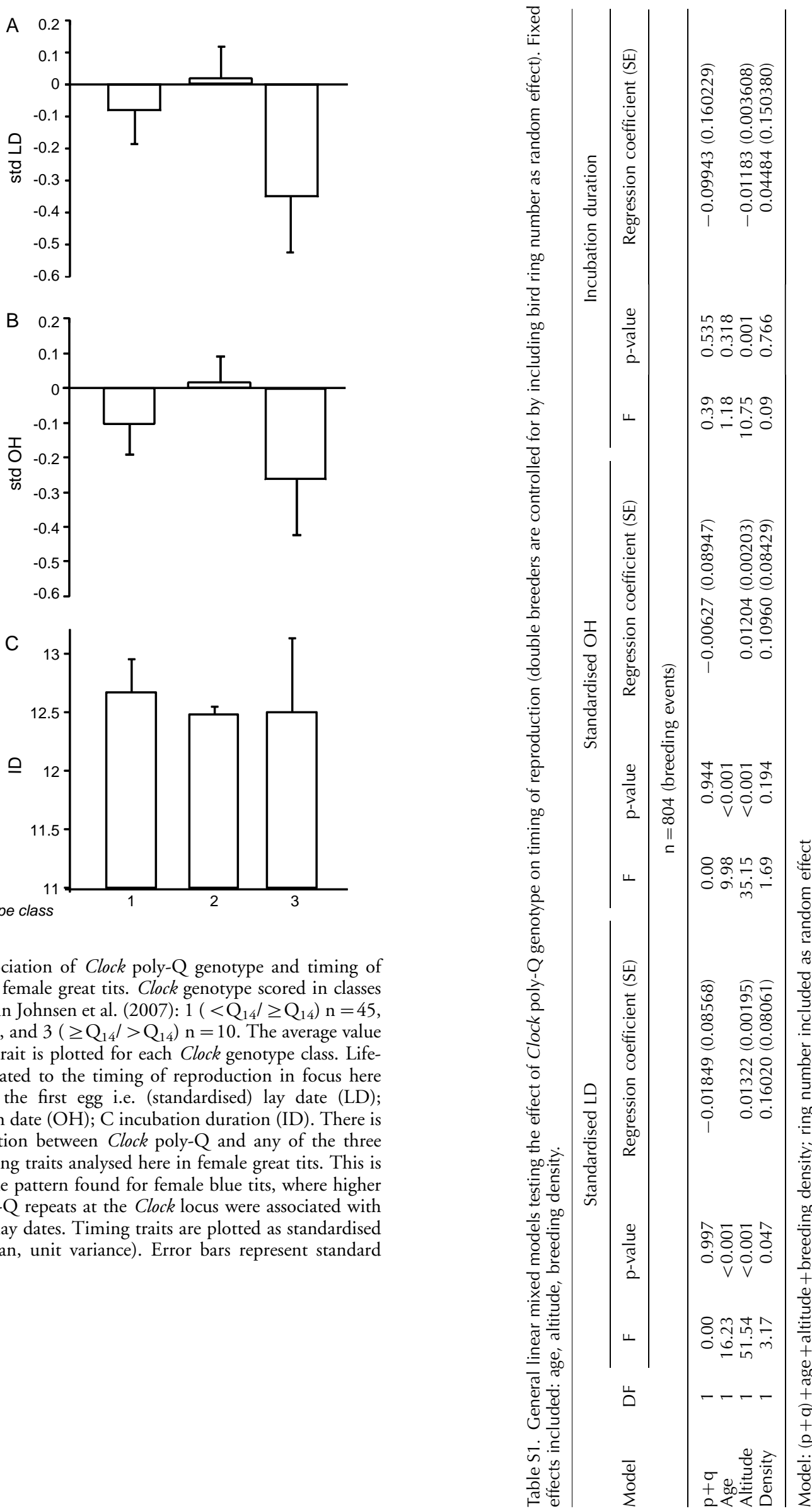

Figure S1. Association of Clock poly-Q genotype and timing of reproduction in female great tits. Clock genotype scored in classes similar to those in Johnsen et al. (2007): $1\left(<\mathrm{Q}_{14} / \geq \mathrm{Q}_{14}\right) \mathrm{n}=45$, $2\left(Q_{14}\right) n=749$, and $3\left(\geq Q_{14} />Q_{14}\right) n=10$. The average value of each timing trait is plotted for each Clock genotype class. Lifehistory traits related to the timing of reproduction in focus here are: A date of the first egg i.e. (standardised) lay date (LD); $\mathrm{B}$ observed hatch date $(\mathrm{OH}) ; \mathrm{C}$ incubation duration (ID). There is no clear association between Clock poly-Q and any of the three timing of breeding traits analysed here in female great tits. This is in contrast to the pattern found for female blue tits, where higher number of poly-Q repeats at the Clock locus were associated with later hatch and lay dates. Timing traits are plotted as standardised values (zero mean, unit variance). Error bars represent standard errors. 


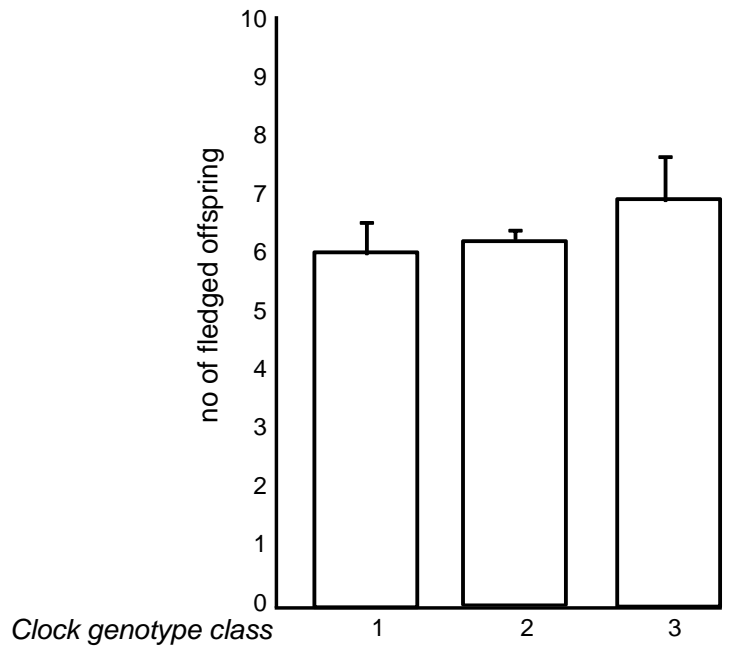

Figure S2. Association between Clock poly-Q genotype (genotype classes as in Fig. S1) and reproductive success in blue tit females calculated as average number of fledged offspring. Our results show no clear effect of Clock poly-Q allele length on the number of offspring successfully raised to the fledging stage. Error bars represent standard errors.
Table S2. GLMM testing for an effect of Clock poly-Q genotype (standardised values for $\mathrm{p}+\mathrm{q}$ ) on reproductive success (shown for number of fledged offspring, also tested for number of total recruits and number of total grand recruits (tested separately for grand recruits via daughters or sons, and total grandrecruits), all n.s.). Fixed effects included are: age, year of breeding. Ring number included as random effect.

\begin{tabular}{llrrrr} 
& \multicolumn{5}{c}{804 breeding events, 521 females } \\
\cline { 2 - 6 } Model & $\mathrm{n}$ & DF & $\mathrm{F}$ & $\mathrm{p}$ & \multicolumn{1}{c}{$\begin{array}{c}\text { Regression } \\
\text { coefficient (SE) }\end{array}$} \\
\hline$(\mathrm{p}+\mathrm{q})_{\text {stand }}$ & 804 & 1 & 1.11 & 0.293 & $0.2438(0.24761)$ \\
$\left.(\mathrm{p}+\mathrm{q})_{\text {stand }}\right)^{2}$ & & 1 & 0.29 & 0.588 & $0.0270(0.04976)$ \\
Age & 1 & 1.12 & 0.308 & \\
Year & & 1 & 15.14 & $<0.001$ & \\
\hline
\end{tabular}

